\title{
Structural Dependent Ferromagnetic-Nonmagnetic Phase Change in FePtRu Films
}

\author{
Takashi Hasegawa and Kosuke Ito \\ Department of Materials Science, Akita University, 1-1 Tegata Gakuen-machi, Akita 010-8502, Japan \\ Correspondence should be addressed to Takashi Hasegawa; takashi@gipc.akita-u.ac.jp
}

Received 22 March 2017; Revised 25 May 2017; Accepted 4 June 2017; Published 4 July 2017

Academic Editor: Gianluca Gubbiotti

Copyright (C) 2017 Takashi Hasegawa and Kosuke Ito. This is an open access article distributed under the Creative Commons Attribution License, which permits unrestricted use, distribution, and reproduction in any medium, provided the original work is properly cited.

\begin{abstract}
Herein, we studied correlations between crystal structures and magnetic properties of $\mathrm{FePt}_{1-x} \mathrm{Ru}_{x}$ films. At room temperature, the chemical disordered $A 1$ films with $0 \leq x<0.20$ and $0.20 \leq x \leq 1.00$ exhibited ferromagnetic properties and paramagnetic properties, respectively. Curie temperature of the disordered film with $x=0.30$ was $200 \mathrm{~K}$. In contrast, the ordered $L 1_{0}$ films had ferromagnetic properties in a wider range of $0 \leq x<0.80$ with the magnetic easy-axis perpendicular to the film plane. For $0 \leq x \leq 0.50$, with the ordered structure, the films had high magnetization and high uniaxial magnetic anisotropy of over $10^{7} \mathrm{erg} \mathrm{cm}^{-3}$. For $x=0.60-0.70$ with the ordered structure, a temperature-dependent magnetic phase transition appeared, and magnetization reached its maximum value at around $200 \mathrm{~K}$. Using this material system, we proposed a nanopatterning method involving a ferromagnetic-paramagnetic phase change due to the ordered-disordered structural transformation.
\end{abstract}

\section{Introduction}

Increased power consumption in data centers having millions of hard disk drives (HDDs) has emerged as a serious issue. Decreasing the number of HDDs by increasing their magnetic storage density can be a simple and efficient means to mitigate increased power consumption. Ferromagnetic (FM) thin films prepared using nanofabrication techniques can be used for high-density magnetic data storage [1-3] and spintronic devices [4].

Bit-patterned media (BPM) is a candidate for the creation of ultra-high-density magnetic data storage devices $[1,2]$. BPM consist of multiple phases, which include the FM phase (dots) characterized by a high uniaxial magnetic anisotropy along with, in principle, diamagnetic, antiferromagnetic (AF), and paramagnetic (PM) phases. Ion milling is conventionally used for nanofabrication of two-dimensional FM films [5, 6], which produces physically separated FM regions. After ion milling, backfill and polishing stages are required to obtain BPM with smooth surfaces, which is a prerequisite for read/write heads to be able to fly at a few nanometers above the medium surface [1].
Another BPM fabrication method, using ion irradiation, has also been proposed $[7,8]$. This method can replace the three processes of ion milling, backfill, and polishing steps, thereby streamlining the production of BPM. Ion irradiation results in structural disordering. $\mathrm{Co} / \mathrm{Pt}$ multilayers $[7,8]$ and $\mathrm{L1}_{0} \mathrm{CrPt}_{3}$ films [9] with an uniaxial magnetocrystalline anisotropy constant $\left(K_{u}\right)$ of $\sim 10^{6} \mathrm{erg} \mathrm{cm}^{-3}$, which is not sufficient for the realization of high magnetic data storage density of over $2 \mathrm{~Tb} \mathrm{in}^{-2}$, are transformed from FM to PM phase after ion irradiation.

A problem faced by high-density magnetic data storage is the thermal fluctuation effect of magnetic grains or dots. The magnetic anisotropy energy $\left(K_{u} V ; V\right.$ : volume of an isolated magnetic grain or dot) becomes lower relative to the thermal fluctuation energy $\left(k_{B} T ; k_{B}\right.$ : Boltzmann constant, $T$ : temperature) when the grain and/or dot size is reduced for increasing storage density. The thermal fluctuation can be sufficiently reduced using materials with high $K_{u}$, satisfying the thermal stability factor requirement of $\left(K_{u} V / k_{B} T\right)>60$ (this metric was derived using the Sharrock equation [10]) which is typically regarded as a minimum requirement for magnetic data storage $[1,11]$. 
$L 1_{0}$ FePt (CuAu-I type, fct, $a=b>c$ ) films with ordered structures are possible candidates for BPM due to their high $K_{u}$ of $7.0 \times 10^{7} \mathrm{erg} \mathrm{cm}^{-3}$, high saturation magnetization $\left(M_{s}\right)$ of $1100 \mathrm{emu} \mathrm{cm}^{-3}$, high corrosion resistance, and low resistivity [12-14], which leads to excellent thermal stability of magnetization in nanometer-size structures. After ion irradiation, the ordered $L 1_{0}$ FePt films undergo transformation from the hard-FM phase (high $K_{u}$ ) to the soft-FM phase (low $K_{u}$ ) with the disordered $A 1$ structure (fcc, $a=$ $b=c)$ [15-20]. Although the $L 1_{0}$ FePt is suitable for BPM, modifying its properties using ion irradiation is difficult due to the insensitivity of magnetization with ion irradiation. The high spontaneous magnetization $\left(M_{0}\right)$ of the soft-FM region (interdot spacing), wherein the ions are irradiated, leads to spike noise in the storage media. Therefore, developing high$K_{u}$ and high- $M_{s}$ materials, whose $K_{u}$ and $M_{s}$ values decrease upon ion irradiation with high sensitivity, is required.

In our previous study, nonmagnetic phases were observed by replacing Fe with $\mathrm{Mn}$ [21] and Pt with $\mathrm{Rh}$ [22] in FePt films, and the former improved the sensitivity of $M_{s}$ to ion irradiation. The $L 1_{0} \mathrm{Fe}_{1-x} \mathrm{Mn}_{x} \mathrm{Pt}$ films with $x \leq 0.44$ exhibit FM properties corresponding to $K_{u}>2.1 \times 10^{7} \mathrm{erg} \mathrm{cm}^{-3}$, whereas, disordered $A 1$ films with $x \geq 0.44$ possess PM properties at room temperature. These films change from a FM to PM state as the $L 1_{0}$ structure transforms into the $A 1$ structure due to ion irradiation. However, the high $\mathrm{Mn}$ contents could decay corrosion resistance and $K_{u}$. By replacing $\mathrm{Pt}$ with $\mathrm{Ru}$ in the $L 1_{0}$ FePt films, which could be uninfluential to its corrosion resistance, a reduction of Curie temperature $\left(T_{c}\right)$ has been reported [23].

In this study, by replacing $\mathrm{Pt}$ with $\mathrm{Ru}$ in $A 1$ and $L 1_{0} \mathrm{FePt}$ films, correlations between crystal structures and magnetic properties were investigated.

\section{Materials and Methods}

$\mathrm{Fe}_{50}\left(\mathrm{Pt}_{1-x} \mathrm{Ru}_{x}\right)_{50}$ films with the thickness of $6.0 \mathrm{~nm}$ were deposited by magnetron cosputtering at a base pressure of $10^{-5} \mathrm{~Pa}$ using $\mathrm{Ar}$ gas at $0.5 \mathrm{~Pa}$ on a single-crystalline $\mathrm{MgO}(100)$ substrate at $298 \mathrm{~K}$. The $\mathrm{Ru}$ composition $(x)$ was controlled by varying the sputtering rates of the $\mathrm{Pt}$ and $\mathrm{Ru}$ targets and was detected using an electron probe X-ray microanalyzer. The films were annealed by rapid thermal annealing (RTA) with a heating rate of $300 \mathrm{~K} \mathrm{~s}^{-1}$ under a vacuum of $2 \times 10^{-4} \mathrm{~Pa}$. The crystalline structure was studied using out-of-plane X-ray diffraction (XRD) measurement and in-plane XRD measurement with $\mathrm{CuK} \alpha$ radiation. A vibrating sample magnetometer (VSM) with a maximum field of $18 \mathrm{kOe}$ and a superconducting quantum interference device (SQUID) magnetometer with a maximum field of $50 \mathrm{kOe}$ were used to assess the magnetic properties of the films.

\section{Results and Discussion}

Figure 1(a) shows out-of-plane XRD patterns for the asdeposited $\mathrm{FePt}_{1-x} \mathrm{Ru}_{x}$ films of thickness $6.0 \mathrm{~nm}$. The $\mathrm{Ru}$ composition $x$ of the $\mathrm{FePt}_{1-x} \mathrm{Ru}_{x}$ films was changed from 0 to 1.00 and is shown from 0 to 0.30 with the background pattern (sample holder and substrate) in the figure. Only the background peaks were observed for all the films, since the fundamental (111) reflection peaks for the disordered $A 1$ structure appear at the same angle as the $\mathrm{MgO}(100)$ substrate.

Figures 1(b)-1(e) show magnetization curves of the films with $x=0,0.10,0.20$, and 0.30 , measured by the VSM with a maximum field of $18 \mathrm{kOe}$ applied perpendicular $(\perp$, dashed line) and parallel (//, solid line) to the film plane at $298 \mathrm{~K}$. The magnetic easy-axes of all the films are parallel to the film plane. Figure 1(f) shows the temperature $(T)$ dependence of magnetization $(M)(M-T$ curve) for the film with $x=0.30$ at temperatures at or below room temperature $(300 \mathrm{~K}$ down to $30 \mathrm{~K})$, measured using a SQUID magnetometer with a field of $1 \mathrm{kOe}$ applied parallel to the film plane after saturating the magnetization by a field of $50 \mathrm{kOe}$ at $298 \mathrm{~K}$. $M$ decreases with an increase in $T$ and approaches 0 at around $T_{c}$ of $200 \mathrm{~K}$. $T_{c}$ was estimated from the $M^{2}-T$ curve (not shown). This indicates that the film with $x=0.30$ is in the PM phase at room temperature.

The $x$-dependence of $M_{0}$, determined using the Arrott plot [24] at $298 \mathrm{~K}$, is shown in Figure $1(\mathrm{~g}) . M_{0}$ decreases with increasing $x$, and an abrupt decrease in $M_{0}$ is found at $x=0.20$, caused by the decrease in $T_{c}$. This implies that the films with $0 \leq x<0.20$ are in the FM phase, and the films with $x \geq 0.20$ are in the PM phase at room temperature.

Figures 2(a) and 2(b) show out-of-plane XRD patterns and in-plane XRD patterns, respectively, for the $\mathrm{FePt}_{1-x} \mathrm{Ru}_{x}$ films after annealing at $1173 \mathrm{~K}$ for $4 \mathrm{~h}$ with a heating rate of $300 \mathrm{~K} \mathrm{~s}^{-1}$ by RTA. In Figure 2(a), only superlattice (001) and fundamental (002) reflection peaks for the ordered $L 1_{0}$ structure are observed for the films with $0 \leq x \leq 0.70$, whereas only background peaks were observed for the films with $0.80 \leq x \leq 1.00$ due to overlapping of the fundamental (111) reflection peak for the disordered $A 1$ structure and the reflection peaks for the $\mathrm{MgO}$ (100) substrate. In Figure 2(b), only the fundamental (200) reflection peak is observed for the films with $0 \leq x \leq 0.60$, whereas only background peaks are observed for the films with $0.70 \leq x \leq 1.00$. These reflection patterns indicate that the (001) crystalline texture is normal to the film plane in the films with $0 \leq x \leq 0.70$, whereas the disordered $A 1$ structure is obtained in the films with $0.80 \leq x \leq 1.00$. This result is broadly consistent with the previous report [23]. The degrees of long-range chemical order parameter were estimated to be $\sim 0.90$ in the films with $0 \leq x \leq 0.60$.

The lattice constants $(a, c$, and $c / a)$ are plotted as functions of $x$ in Figure 2(c). $c$ and $a$ values were obtained from the out-of-plane and in-plane XRD measurements, respectively. $a$ and $c$ values of the film with $x=0$ are about $0.3867 \mathrm{~nm}$ and $0.3726 \mathrm{~nm}$, respectively, and are close to those of the FePt bulk alloy ( $a=0.3852 \mathrm{~nm}, c=0.3713 \mathrm{~nm}$; PDF number 43-1359). The values of $a$ are $\sim 0.39 \mathrm{~nm}$ for each $x$ which could be kept by the epitaxial growth, whereas $c$ and the axial ratio $(c / a)$ values decrease with increasing $x$. By considering the layerby-layer atomic configuration in $L 1_{0} \mathrm{FePt}$ and the differences of atomic radiuses of $\mathrm{Pt}, \mathrm{Ru}$, and $\mathrm{Fe}(\mathrm{Pt}>\mathrm{Ru}>\mathrm{Fe})$, the 


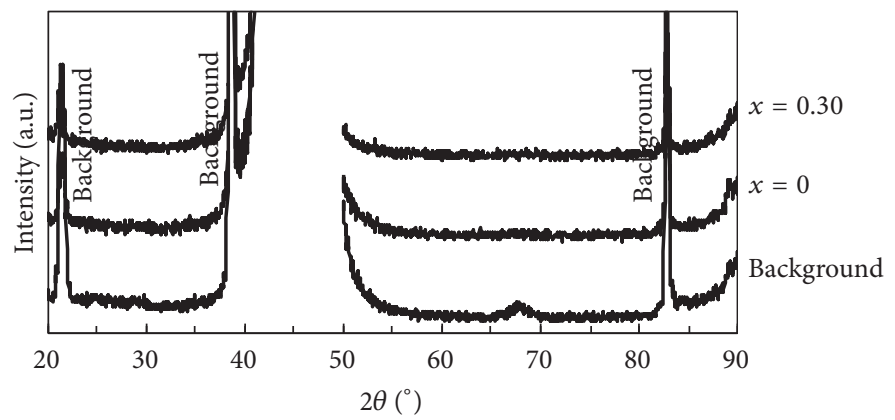

(a)

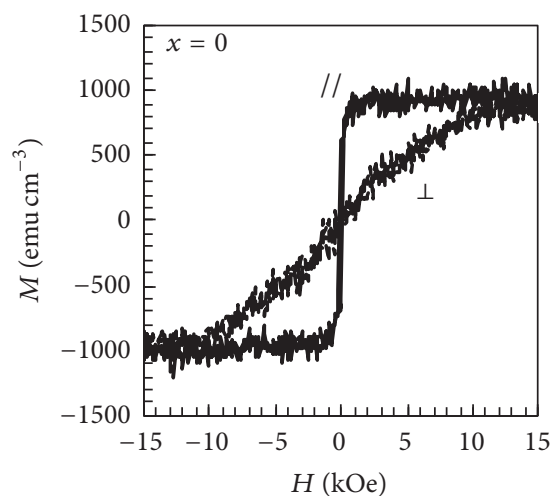

(b)

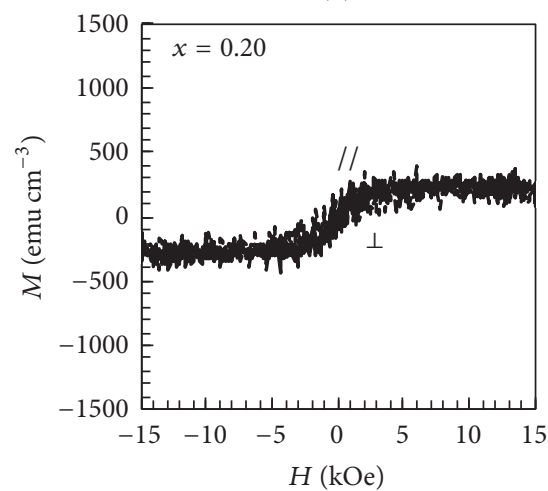

(d)

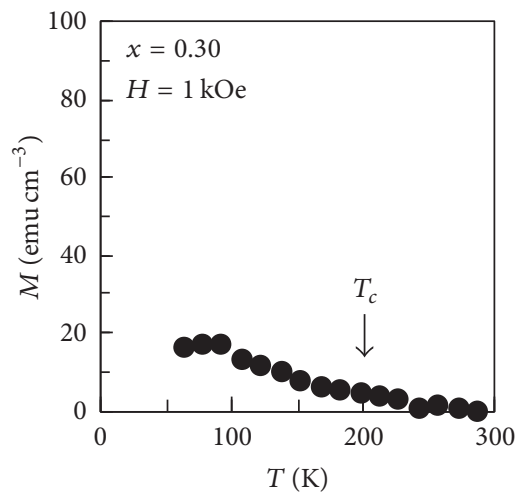

(f)

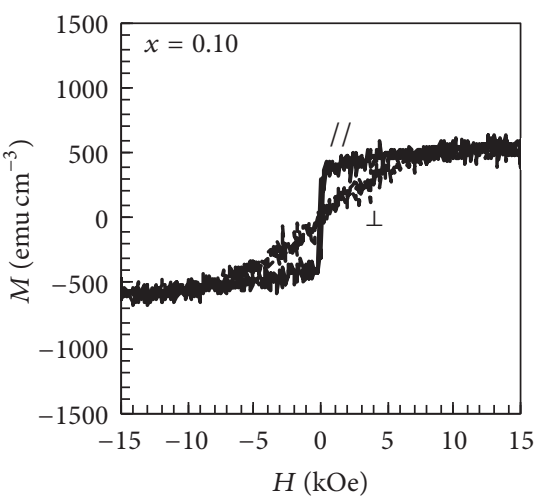

(c)

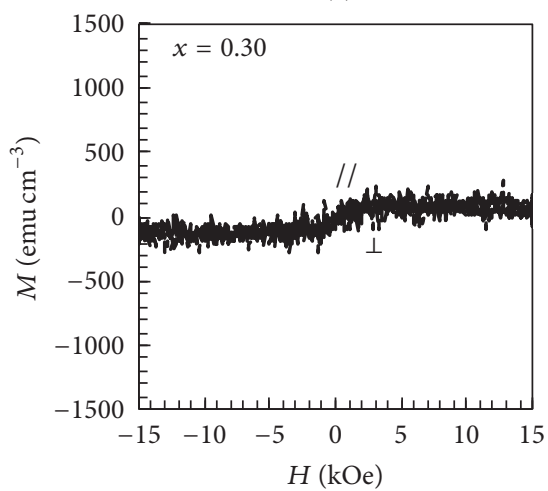

(e)

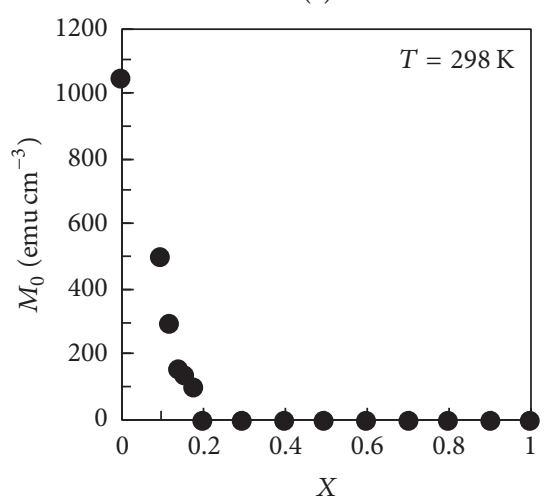

(g)

Figure 1: Crystal structure and magnetic properties of disordered $A 1 \mathrm{FePt}_{1-x} \mathrm{Ru}_{x}$ films before annealing (as-deposited films). (a) Out-of-plane XRD patterns. Magnetization curves of the films with $x=$ (b) 0 , (c) 0.10 , (d) 0.20 , and (e) 0.30 . Magnetic fields $(H)$ were applied perpendicular ( $\perp$, dashed line) and parallel (//, solid line) to the film plane at $298 \mathrm{~K}$. (f) Temperature dependence of magnetization $(M-T$ curve) for the film with $x=0.30$, measured from $300 \mathrm{~K}$ to $30 \mathrm{~K}$ with a field of $1 \mathrm{kOe}$ applied parallel to the film plane after saturation. (g) $x$-dependence of $M_{0}$ at $298 \mathrm{~K}$. 


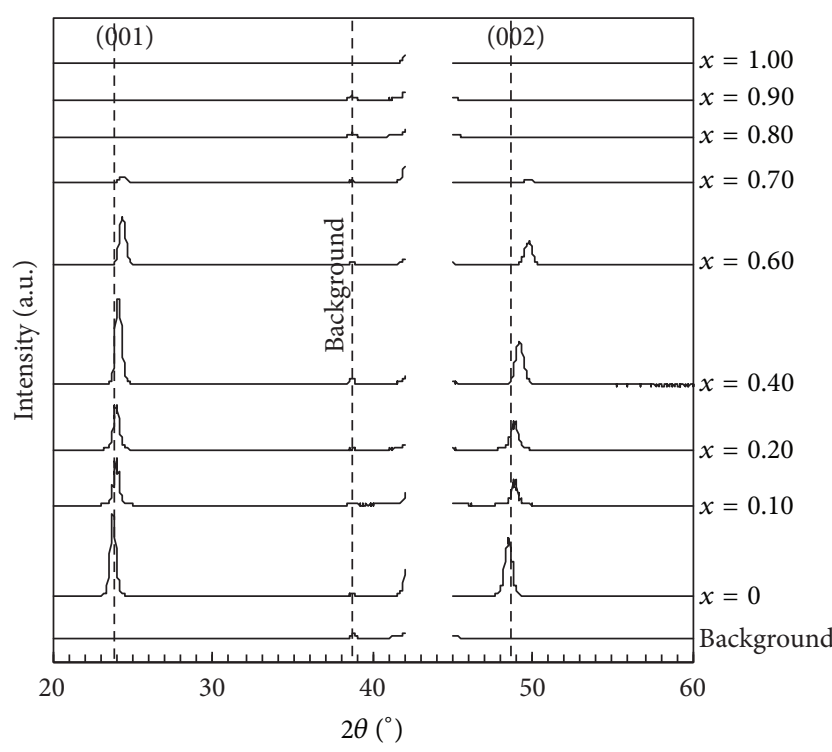

(a)

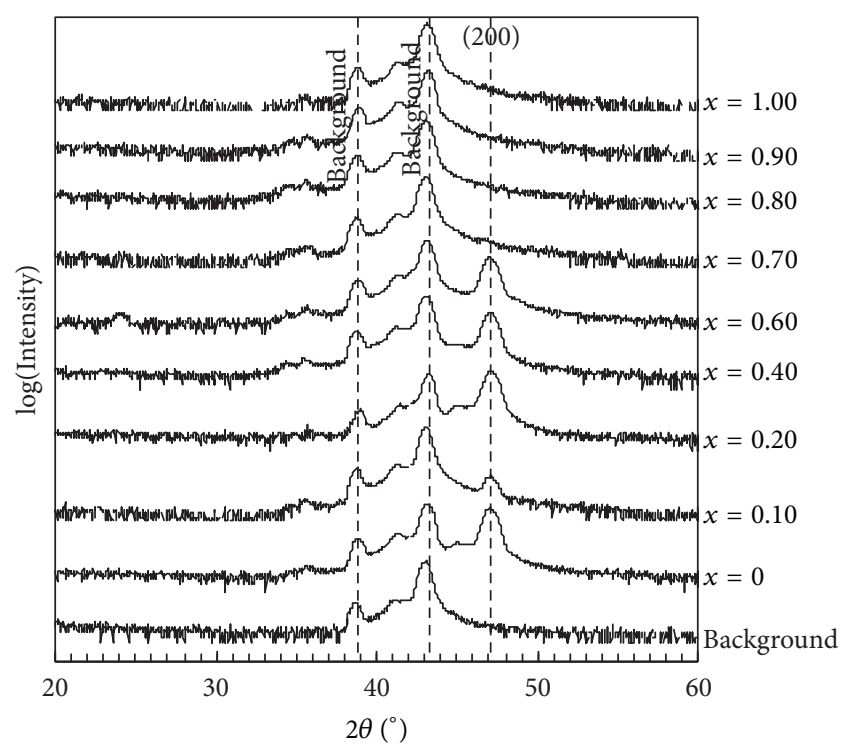

(b)
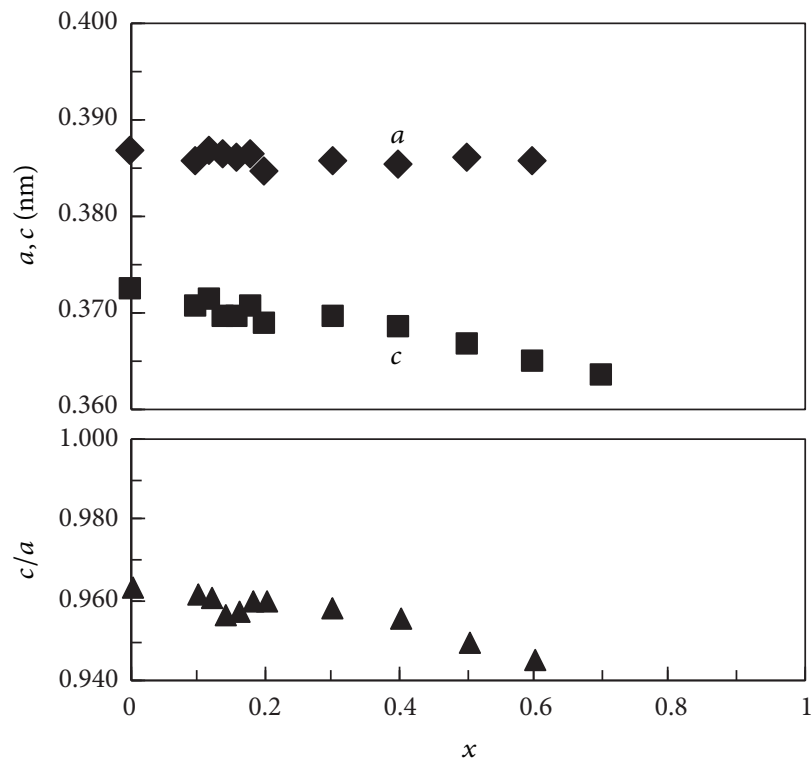

(c)

FIGURE 2: Crystal structure of $\mathrm{FePt}_{1-x} \mathrm{Ru}_{x}$ films after annealing at $1173 \mathrm{~K}$ for $4 \mathrm{~h}$. (a) Out-of-plane XRD patterns and (b) in-plane XRD patterns. (c) Lattice constants $(a, c$, and $c / a)$ as a function of $x$.

decrease in the lattice constant $c$ indicates the substitution of $\mathrm{Ru}$ for Pt.

Figures 3(a)-3(e) show magnetization curves of the films with $x=0,0.10,0.20,0.40$, and 0.60 , measured by the SQUID magnetometer with a maximum field of $50 \mathrm{kOe}$ applied perpendicular $(\perp$, filled symbols) and parallel $(/ /$, open symbols) to the film plane at $298 \mathrm{~K}$. The magnetic easyaxes of all the films were perpendicular to the film plane. $K_{u}$ values were evaluated using the magnetization curves [25]. The magnetic anisotropy field $\left(H_{k}\right)$ was determined from the intersection point of the extrapolated magnetization curves of the magnetic fields applied parallel and perpendicular to the film plane. Only the linear part of the in-plane magnetization curve was extrapolated. Consequently, $K_{u}$ was obtained using the relation $K_{u}=\left(M_{s} \times H_{k} / 2\right)+K_{\text {shape}}$, where $K_{\text {shape }}$ is the shape anisotropy calculated using the demagnetization factors $(N)$ of the film shape $\left(N_{\perp}=4 \pi, N_{/ /}=0\right)$. The films with $0 \leq x \leq 0.50$ have high magnetization of $500 \leq M_{0} \leq$ $800 \mathrm{emu} \mathrm{cm}^{-3}$, high coercivity of $15 \leq H_{c} \leq 43 \mathrm{kOe}$, and high anisotropy of $2.6 \times 10^{7} \leq K_{u} \leq 5.0 \times 10^{7} \mathrm{erg} \mathrm{cm}^{-3}$ in the perpendicular direction, and these results are broadly consistent with the previous report [23].

The $x$-dependence of $M_{0}$ and $K_{u}$ is plotted in Figure 3(f). $M_{0}$ and $K_{u}$ decrease with increasing $x$ and reach almost 0 at $x=0.80$, which is the critical composition for the transition from the $L 1_{0}$ structure to the $A 1$ structure. For instance, $L 1_{0}$ 


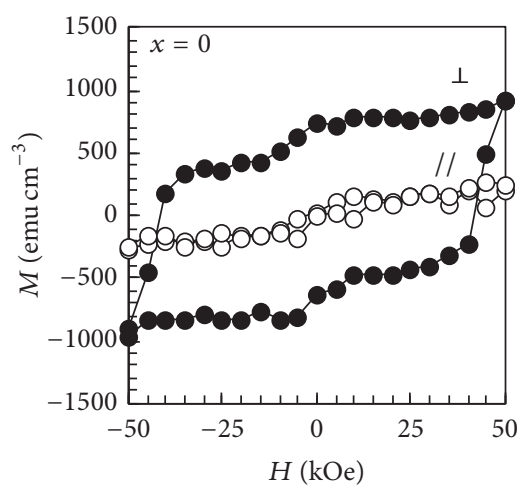

(a)

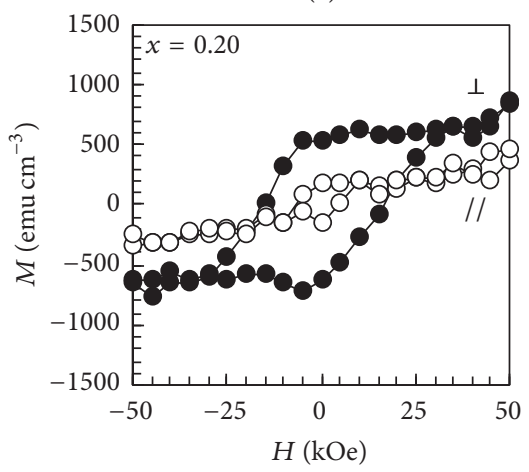

(c)

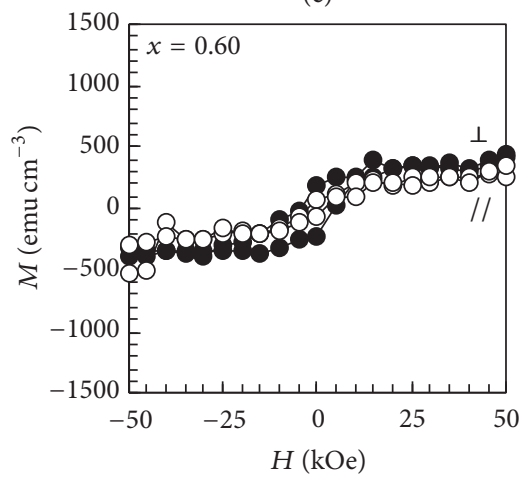

(e)

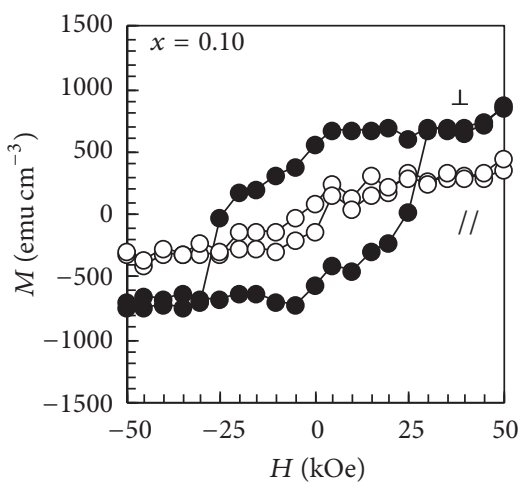

(b)

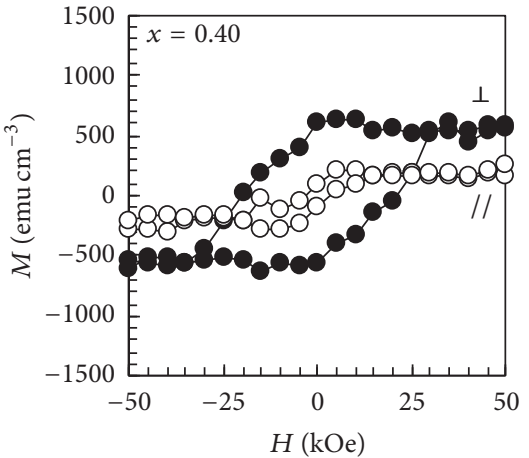

(d)

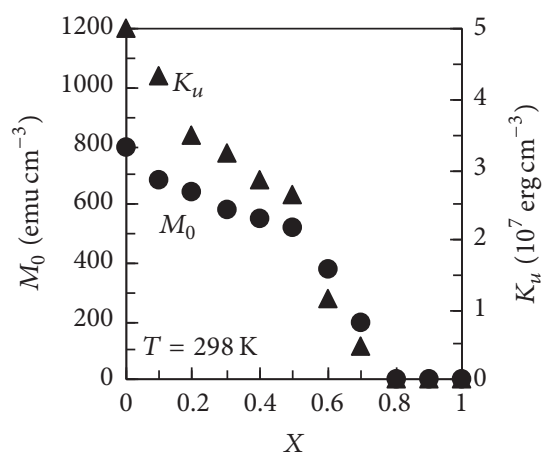

(f)

Figure 3: Magnetic properties of the $\mathrm{FePt}_{1-x} \mathrm{Ru}_{x}$ films after annealing at $1173 \mathrm{~K}$ for $4 \mathrm{~h}$. Magnetization curves of the ordered $L 1_{0} \mathrm{FePt}_{1-x} \mathrm{Ru}_{x}$ films with $x=$ (a) 0 , (b) 0.10 , (c) 0.20 , (d) 0.40 , and (e) 0.60 . $H$ was applied perpendicular ( $\perp$, filled symbols) and parallel (//, open symbols) to the film plane at $298 \mathrm{~K}$. (f) $x$-dependence of $M_{0}$ and $K_{u}$ at $298 \mathrm{~K}$.

film with $x=0.20$ has FM properties with a $K_{u}=3.5 \times$ $10^{7} \mathrm{erg} \mathrm{cm}^{-3}$, whereas, the disordered $A 1$ film with $x=0.20$ has PM properties at room temperature. The film can change from a FM to PM state as the $L 1_{0}$ structure transforms into the $A 1$ structure due to ion irradiation. By using the thermal stability factor $\left(K_{u} V / k_{B} T\right)>60$ and $K_{u}=3.5 \times 10^{7} \mathrm{erg} \mathrm{cm}^{-3}$, the smallest diameter of the spherical shape was estimated to be $\sim 5.1 \mathrm{~nm}$, which indicates that this material system has sufficient thermal stability for use in high-density magnetic data storage media of $\sim 10 \mathrm{~Tb}^{-2}$.

Figures 4(a)-4(d) show the $M-T$ curves of the films with $x=0.20,0.40,0.60$, and 0.80 at temperatures at or below room temperature $(300 \mathrm{~K}$ down to $30 \mathrm{~K})$, measured using the SQUID magnetometer with a field of $1 \mathrm{kOe}$ applied perpendicular to the film plane after saturating the magnetization by a field of $50 \mathrm{kOe}$ at $298 \mathrm{~K}$. In the films with $x \leq 0.40, M$ increases with a decrease in $T$ due to the typical FM properties. However, at $x=0.60, M$ reaches its maximum value at $210 \mathrm{~K}\left(T_{0}\right)$ and decreases with a decrease in temperature below $T_{0}$. This indicates that the film with $x=0.60$ can contain an antiferromagnetically ordered phase (spin-glass or canted-AF etc.) at temperatures below $T_{0}$. In the films with $x \geq 0.80, M$ is almost 0 at each $T$. These results imply that the films with $0 \leq x \leq 0.40$ should have the FM-PM transition above $300 \mathrm{~K}$, the films with $0.40<x<$ 0.80 have the antiferromagnetically ordered phase FM-PM transition, and the films with $0.80 \leq x \leq 1.00$ have the FM$\mathrm{PM}$ transition below $300 \mathrm{~K}$ due to the $A 1$ structure. 


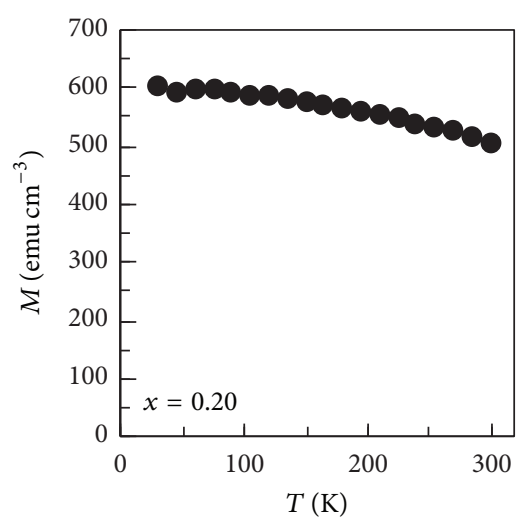

(a)

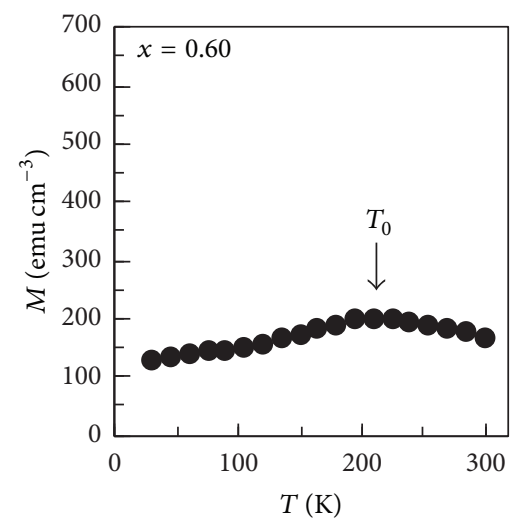

(c)

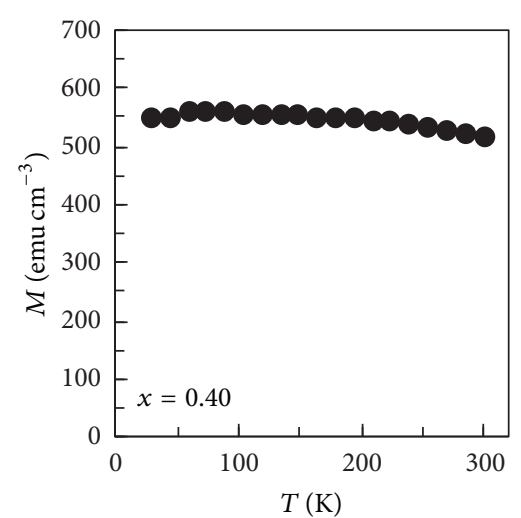

(b)

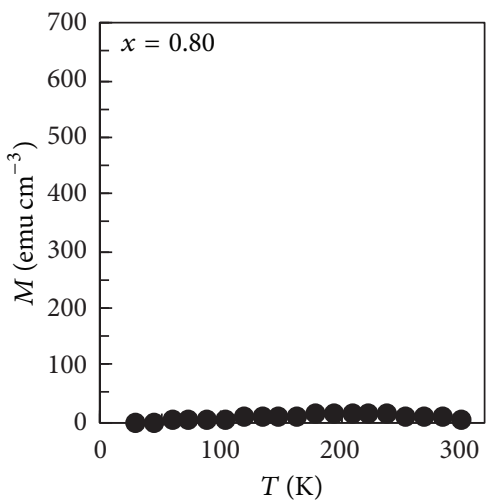

(d)

FiguRE 4: Temperature dependence of magnetization ( $M$-T curves) for the $\mathrm{FePt}_{1-x} \mathrm{Ru}_{x}$ films after annealing at $1173 \mathrm{~K}$ for $4 \mathrm{~h}$ with $x=(\mathrm{a}) 0.20$, (b) 0.40 , (c) 0.60 , and (e) 0.80 , measured from $300 \mathrm{~K}$ to $30 \mathrm{~K}$ with a field of $1 \mathrm{kOe}$ applied perpendicular to the film plane after saturating.

Finally, we propose a nanopatterning method for this material system. According to our previous results, the ordered $L 1_{0}$ structures of FePtX were easily transformed to the disordered $A 1$ structure under ion irradiation [15-21]. Figure 5 shows the schematics of a nanopatterning method using an FM-PM phase change due to the $L 1_{0}-A 1$ structural transformation caused by ion irradiation. After ion irradiation, FM dots having $L 1_{0}$ structure should be surrounded by PM spacing having $A 1$ structure with a smooth disc surface.

\section{Conclusions}

Correlations between crystal structures and magnetic properties of $\mathrm{FePt}_{1-x} \mathrm{Ru}_{x}$ films (6.0 nm thick) were investigated.

(1) Magnetic properties of $A 1$ disordered structure (asdeposited films): $M_{0}$ decreased with increasing $x$, and an abrupt decrease in $M_{0}$ was found at $x=0.20$ at room temperature, due to the decrease in $T_{c}$. In the range of $0 \leq$ $x<0.20$, the films had FM properties $\left(100 \leq M_{0} \leq\right.$ $1050 \mathrm{emu} \mathrm{cm}^{-3}$ ) with their magnetic easy-axes parallel to the film plane. In the range of $x \geq 0.20$, the films had PM properties $\left(M_{0} \approx 0 \mathrm{emu} \mathrm{cm}^{-3}\right)$ at room temperature.

(2) Magnetic properties of $L 1_{0}$ ordered structure (annealed films): $M_{0}$ and $K_{u}$ decreased with increasing $x$. In the range of $0 \leq x \leq 0.50$, the films had FM properties
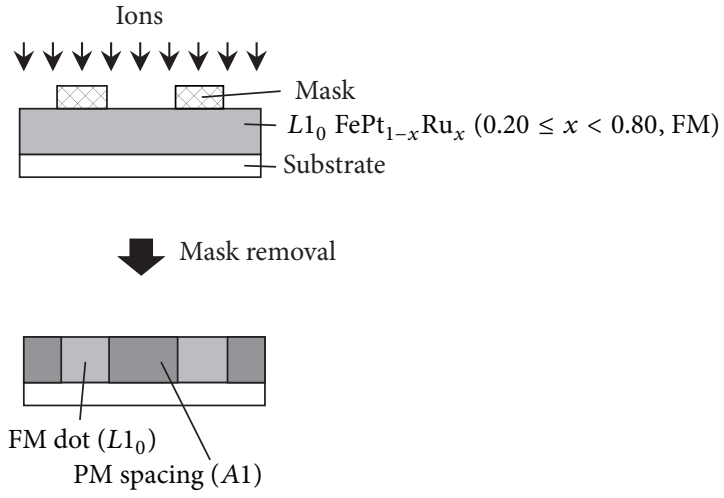

FIGURE 5: Schematics of the nanopatterning method using FM-PM phase change.

$\left(500 \leq M_{0} \leq 800 \mathrm{emu} \mathrm{cm}^{-3}, 15 \leq H_{c} \leq 43 \mathrm{kOe}, 2.6 \times 10^{7} \leq\right.$ $K_{u} \leq 5.0 \times 10^{7} \mathrm{erg} \mathrm{cm}^{-3}$ ), with their magnetic easy-axis perpendicular to the film plane. In the range of $0.40<x<$ 0.80 , the films could contain an antiferromagnetically ordered phase at temperatures below room temperature.

For instance, the $L 1_{0}$ film with $x=0.20$ had FM properties with a $K_{u}=3.5 \times 10^{7} \mathrm{erg} \mathrm{cm}^{-3}$, whereas the disordered $A 1$ film with $x=0.20$ had PM properties at 
room temperature. The film could change from a FM to PM state as the $L 1_{0}$ structure transforms into the $A 1$ structure due to ion irradiation. These results suggest the possibility of applying the material system for nanopatterning method for high-density magnetic storage media.

\section{Conflicts of Interest}

The authors declare that there are no conflicts of interest regarding the publication of this article.

\section{Acknowledgments}

This work was supported by the Japan Society for the Promotion of Science KAKENHI through its Grant-in-Aid for Young Scientists (A) (ID: JP15H05518). A part of this work was performed under the Interuniversity Cooperative Research Program (ID: 16K0099) of the Institute for Materials Research, Tohoku University. The authors thank Editage for English language editing.

\section{References}

[1] B. D. Terris and T. Thomson, "Nanofabricated and selfassembled magnetic structures as data storage media," Journal of Physics D: Applied Physics, vol. 38, no. 12, pp. R199-R222, 2005.

[2] A. Kikitsu, "Prospects for bit patterned media for high-density magnetic recording," Journal of Magnetism and Magnetic Materials, vol. 321, no. 6, pp. 526-530, 2009.

[3] D. Weller, G. Parker, O. Mosendz et al., "Review Article: FePt heat assisted magnetic recording media," Journal of Vacuum Science and Technology B: Nanotechnology and Microelectronics, vol. 34, no. 6, Article ID 060801, 2016.

[4] A. Hirohata, H. Sukegawa, H. Yanagihara et al., "Roadmap for Emerging Materials for Spintronic Device Applications," IEEE Transactions on Magnetics, vol. 51, no. 10, pp. 1-11, 2015.

[5] H. Wang, M. T. Rahman, H. Zhao et al., "Fabrication of FePt type exchange coupled composite bit patterned media by block copolymer lithography," Journal of Applied Physics, vol. 109, no. 7, Article ID 07B754, 2011.

[6] T. Bublat and D. Goll, "Large-area hard magnetic $\mathrm{L1}_{0}-\mathrm{FePt}$ nanopatterns by nanoimprint lithography," Nanotechnology, vol. 22, no. 31, Article ID 315301, 2011.

[7] C. Chappert, H. Bernas, J. Ferré et al., "Planar patterned magnetic media obtained by ion irradiation," Science, vol. 280, no. 5371, pp. 1919-1922, 1998.

[8] B. D. Terris, D. Weller, L. Folks et al., "Patterning magnetic films by ion beam irradiation," Journal of Applied Physics, vol. 87, no. 9, pp. 7004-7006, 2000.

[9] T. Kato, S. Iwata, Y. Yamauchi et al., "Planar patterned media fabricated by ion irradiation into $\mathrm{CrPt}_{3}$ ordered alloy films," Journal of Applied Physics, vol. 105, no. 7, Article ID 07C117, 2009.

[10] M. P. Sharrock, "Time dependence of switching fields in magnetic recording media (invited)," Journal of Applied Physics, vol. 76, no. 10, pp. 6413-6418, 1994.

[11] D. Weller and A. Moser, "Thermal effect limits in ultrahighdensity magnetic recording," IEEE Transactions on Magnetics, vol. 35, no. 6, pp. 4423-4439, 1999.
[12] K. Utsumiya, T. Seki, and K. Takanashi, "Magnetic properties of $\mathrm{L1}_{0}-\mathrm{FePt} /$ permalloy exchange-spring films," Journal of Applied Physics, vol. 110, no. 10, Article ID 103911, 2011.

[13] O. A. Ivanov, L. V. Solina, V. A. Demshina, and L. M. Magat, "Determination of the anisotropy constant and saturation magnetization, and magnetic properties of powders of an ironplatinum alloy," Physics of Metals and Metallography, vol. 35, no. 1, pp. 81-85, 1973.

[14] S. Okamoto, N. Kikuchi, O. Kitakami, T. Miyazaki, Y. Shimada, and K. Fukamichi, "Chemical-order-dependent magnetic anisotropy and exchange stiffness constant of FePt (001) epitaxial films," Physical Review B - Condensed Matter and Materials Physics, vol. 66, no. 2, Article ID 024413, pp. 244131244139, 2002.

[15] T. Hasegawa, G. Q. Li, W. Pei et al., "Structural transition from $\mathrm{L1}_{0}$ phase to A1 phase in FePt films caused by ion irradiation," Journal of Applied Physics, vol. 99, no. 5, Article ID 053505, 2006.

[16] T. Hasegawa, W. Pei, T. Wang et al., "MFM analysis of the magnetization process in $\mathrm{L}_{0}-\mathrm{A} 1 \mathrm{FePt}$ patterned film fabricated by ion irradiation," Acta Materialia, vol. 56, no. 7, pp. 1564-1569, 2008.

[17] T. Hasegawa, T. Yamazaki, and S. Ishio, "Correlation between crystal planes and disordering of ordered $\mathrm{Ll}_{0}$ FePt structure caused by ion irradiation," Materials Letters, vol. 178, pp. 243247, 2016.

[18] N. Gaur, S. Kundu, S. N. Piramanayagam et al., "Lateral displacement induced disorder in $\mathrm{Ll}_{0}-\mathrm{FePt}$ nanostructures by ion-implantation," Scientific Reports, vol. 3, p. 1907, 2013.

[19] S. Kundu, N. Gaur, S. N. Piramanayagam, S. L. Maurer, H. Yang, and C. S. Bhatia, "Ion implantation challenges for patterned media at areal densities over 5 tbpsi," IEEE Transactions on Magnetics, vol. 50, no. 3, pp. 41-46, 2014.

[20] A. Di Bona, P. Luches, F. Albertini et al., "Anisotropy-graded magnetic media obtained by ion irradiation of $\mathrm{Ll}_{0}$ FePt," Acta Materialia, vol. 61, no. 13, pp. 4840-4847, 2013.

[21] T. Hasegawa, R. Kasahara, K. Sasaki, and S. Ishio, "Microfabrication of FeMnPt films involving magnetic phase change due to structural transformation caused by ion irradiation," Physica Status Solidi-Rapid Research Letters, 2016.

[22] T. Hasegawa, J. Miyahara, T. Narisawa et al., "Study of ferroantiferromagnetic transition in [001]-oriented $\mathrm{Ll}_{0} \mathrm{FePt}_{1-x} \mathrm{Rh}_{x}$ film," Journal of Applied Physics, vol. 106, no. 10, Article ID 103928, 2009.

[23] T. Ono, H. Nakata, T. Moriya et al., "Addition of $\mathrm{Ru}$ to $\mathrm{Ll}_{0}-\mathrm{FePt}$ thin film to lower Curie temperature," Applied Physics Express, vol. 9, no. 12, Article ID 123002, 2016.

[24] A. Arrott, "Criterion for ferromagnetism from observations of magnetic isotherms," Physical Review, vol. 108, no. 6, pp. 13941396, 1957.

[25] J. M. Coey, Magnetism and Magnetic Materials, Cambridge University Press, New York, NY, USA, 2010. 

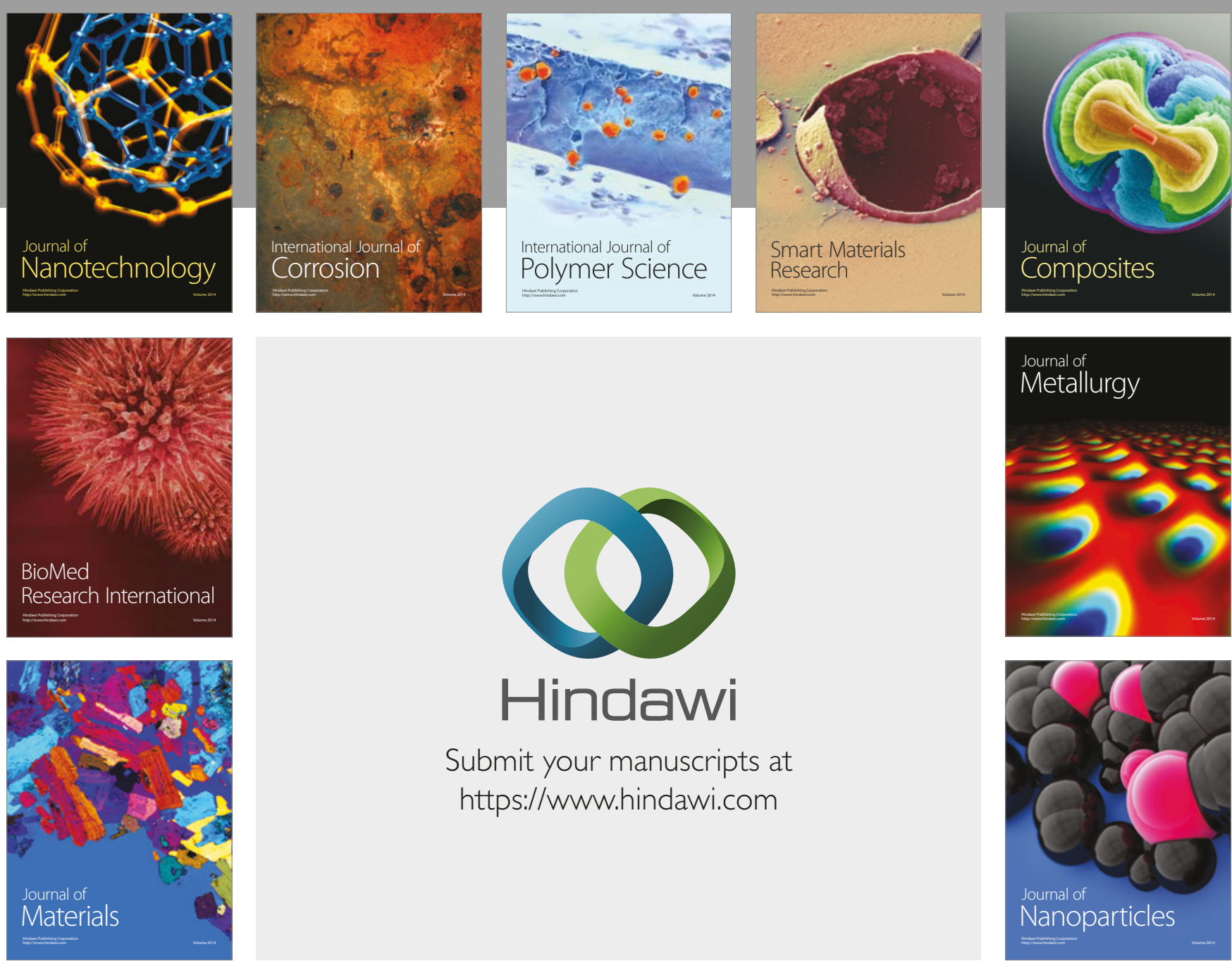

\section{Hindawi}

Submit your manuscripts at

https://www.hindawi.com
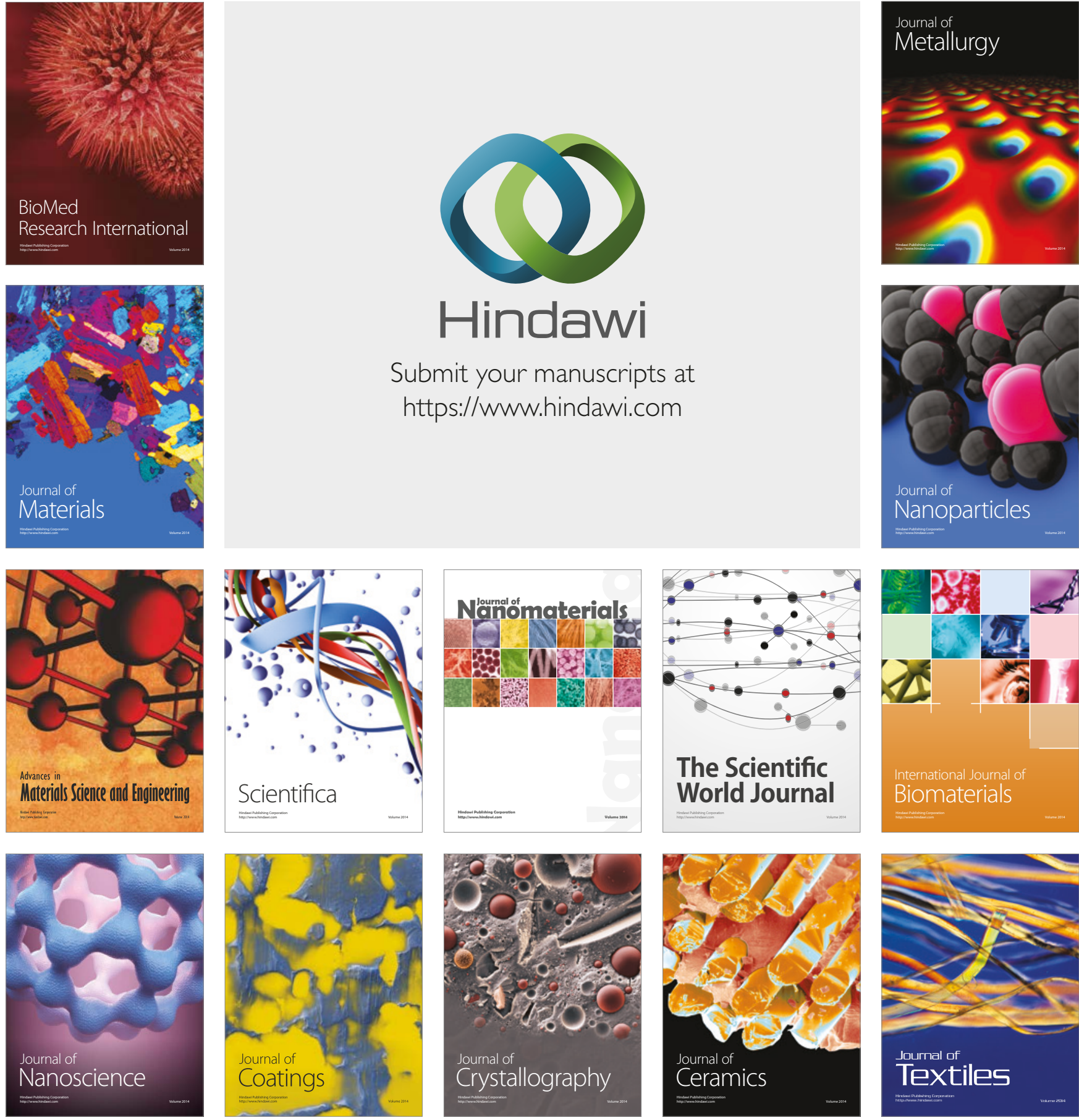

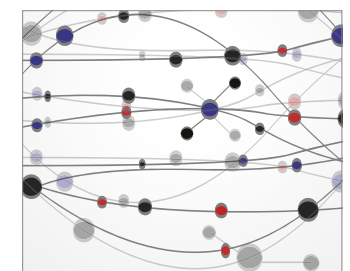

The Scientific World Journal
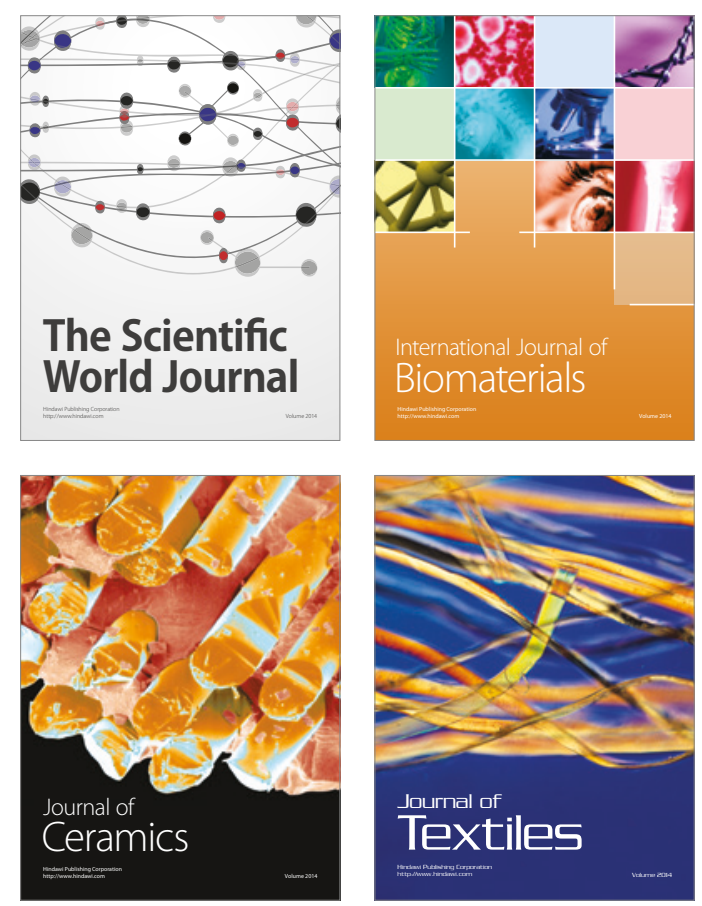